\title{
New development for SuperCam Laser: UV conversion for spectroscopy and downsizing for compact LIBS instrument
}

\begin{abstract}
A. Soujaeff, C. Derycke, S. David, A. Puiseux, Yann Parot, et al.
\end{abstract}

A. Soujaeff, C. Derycke, S. David, A. Puiseux, Yann Parot, E. Durand, B. Faure, M. Boutillier, S. Maurice, "New development for SuperCam Laser: UV conversion for spectroscopy and downsizing for compact LIBS instrument," Proc. SPIE 11852, International Conference on Space Optics - ICSO 2020, 118522P (11 June 2021); doi: 10.1117/12.2599444

SPIE Event: International Conference on Space Optics - ICSO 2021, 2021, Online Only 


\section{International Conference on Space Optics-ICSO 2020}

Virtual Conference

30 March-2 April 2021

Edited by Bruno Cugny, Zoran Sodnik, and Nikos Karafolas
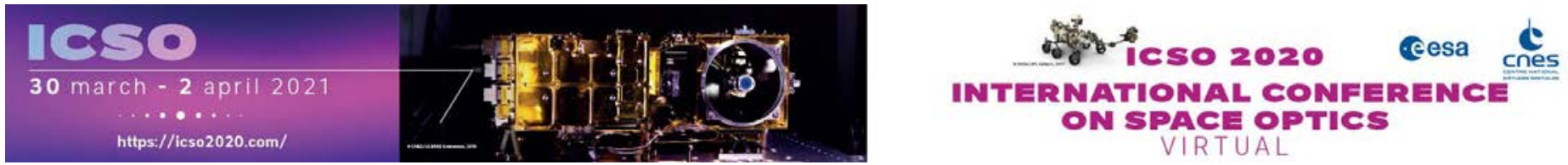

\section{New development for SuperCam Laser: UV conversion for spectroscopy and downsizing for compact LIBS instrument}

\section{Cesa isoporecestings denes}




\title{
New development for SuperCam Laser: UV conversion for spectroscopy and downsizing for compact LIBS instrument
}

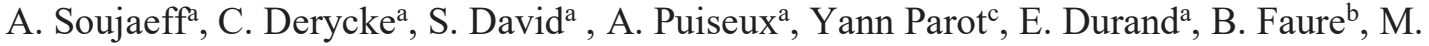 \\ Boutillier $^{\mathrm{b}}$, and S. Maurice ${ }^{\mathrm{c}}$ \\ ${ }^{a}$ THALES LAS France, 2, avenue Gay Lussac, 78995 Elancourt cedex France; ${ }^{b}$ CNES, 18, avenue \\ Edouard Belin, 31401 Toulouse cedex 4, France; 'IRAP, 14, avenue Edouard Belin, 31400 \\ Toulouse, France
}

\begin{abstract}
For planetology applications, a SuperCam type laser emitting in the UV region (266 nm or $355 \mathrm{~nm}$ ) would be of great interest. We investigated Third Harmonic Generation (THG) and Fourth Harmonic Generation (FHG) in LBO crystal and BBO crystal respectively. For potential lunar mission on vehicle smaller than a Curiosity class rover, we designed a laser based on SuperCam heritage focusing on size and mass reduction for a compact LIBS instrument.
\end{abstract}

Keywords: Nd:YAG, LIBS, Raman, Third Harmonic, Fourth Harmonic, Curiosity, Perseverance, SuperCam

\section{INTRODUCTION}

ChemCam is in operation on Mars onboard the Curiosity rover since 2012, with 855,000 shots performed for LIBS analysis [1]. While ChemCam operated only in LIBS mode at its fundamental wavelength (1067 $\mathrm{nm}$ for $\mathrm{Nd}: \mathrm{KGW}$ ), SuperCam can either emit at $1064 \mathrm{~nm}$ for LIBS or $532 \mathrm{~nm}$ for Raman analysis. SuperCam flight model was delivered to CNES in January 2019 and was successfully integrated to Perseverance Rover at NASA JPL. Landing on Mars is scheduled for $18^{\text {th }}$ of February 2021.

Some potential analysis method for planetology would require even shorter wavelength than the second harmonic of $\mathrm{Nd}$ :YAG. Ar/K dating would benefit from the cleaner laser ablation possible with a UV laser. For Raman analysis, it could improve the signal to noise ratio. Laser Induced Fluorescence (LIF) spectroscopy potential has not been proved yet for planetology but would also require a UV laser. For lunar exploration with a rover smaller than Curiosity class, a laser with a reduced footprint would be interesting. This communication describes the harmonic conversion of laser based on the ChemCam and SuperCam heritage as well as the downsizing of SuperCam laser for lunar exploration.

\section{SUPERCAM LASER}

New mission requirements drove the evolution from ChemCam to SuperCam laser. The number of shots in burst mode (from 100 shots to 1000 shots) and the repetition rate (from $3 \mathrm{~Hz}$ to $10 \mathrm{~Hz}$ ) were both increased. A new Raman capability in addition to the LIBS mode required a new shorter wavelength produced using a switchable Second Harmonic Generation (SHG) module. Volume and mass were kept identical to ChemCam. The flight model was delivered to CNES/IRAP in January 2019. It was successfully integrated to NASA rover Perseverance summer 2019. It will land on Mars on 18 February 2021.

\subsection{Oscillator}

The oscillator provides the high beam quality and short pulse length needed for LIBS analysis. It is based on a Nd:YAG rod longitudinally pumped by a multicolor diodes stack. The broad emission spectrum of the diodes shift with 
temperature but always match some absorption peak of the lasing material. This insures pump absorption over large temperature range without the need of any temperature stabilization system for the pumping diodes. Both the diode and the rod are conductively cooled and can operate on a large temperature range $\left(-40^{\circ} \mathrm{C}\right.$ to $40{ }^{\circ} \mathrm{C}$ for qualification).

The oscillator is actively Q-Switched with a RTP Pockels cell to produce nanosecond pulses. Oscillator resonator is linear, closed on one side by the HR coated rod side and by the output coupler on the other side. Reflectivity of the output coupler is $40 \%$. A polarizer, wave-plate and Pockels cell are associated to Q-switch the cavity. The oscillator provides output energy of about $30 \mathrm{~mJ}$ at $1064 \mathrm{~nm}$ with a pulse duration $<5 \mathrm{~ns}$ and a M2 $<2$.

\subsection{Second harmonic generator}

The Second Harmonic Generator (SHG) is a KTP (type II) crystal, placed at the output of the oscillator. It generates the $532 \mathrm{~nm}$ beam used in Raman mode. A RTP electro-optical switch (same Pockels cell as for the oscillator), between the oscillator and SHG, allows the operation mode selection (LIBS or Raman) by switching between linear and circular polarization. In the absence of a high voltage, an infrared beam at $1064 \mathrm{~nm}$ is emitted. When the high voltage is applied to the RTP switch, beam is converted to $532 \mathrm{~nm}$ for Raman analysis. An SHG conversion efficiency of about $50 \%$ is obtained corresponding to about $15 \mathrm{~mJ}$ available for Raman spectroscopy.

\subsection{SuperCam evolutions: Slab amplifiers}

Following the development and manufacturing of the SuperCam model, we studied the possibility to increase further the energy of the laser for both LIBS and Raman applications [2].We added one or two identical slab amplifiers (dimensions $6 \times 6 \times 22 \mathrm{~mm}$ ) to increase the energy at the oscillator output. Each amplifier is transversally pumped by one $1800 \mathrm{~W}$ diode stack identical to the one used for the oscillator. These slab amplifier geometry is similar to the one from ChemCam amplifier with the following modifications:

○ $\mathrm{Nd}: \mathrm{KGW}$ has been replaced by Nd:YAG.

- Two identical multicolor stacks pump the Nd:YAG slab amplifiers to keep running on large temperature range without thermal regulation of the stacks.

Thanks to improved thermal properties of Nd:YAG compared to Nd:KGW, a thousand shot sequence at $10 \mathrm{~Hz}$ can be performed compared to the 100 shots $/ 3 \mathrm{~Hz}$ shooting sequence of ChemCam.

\section{EXPERIMENTAL SET-UP}

\subsection{SuperCam test platform}

We assembled a tabletop version of SuperCam as a flexible platform for low TRL studies (TRL 3-4). We can flexibly add various modules as depicted in figure (1). The oscillator described in paragraph 2.1 is built using optical components from the SuperCam program. As the mechanical mount are off the shelf components, and the system is opened without any covers, it is not representative in term of thermal behavior of the SuperCam Flight model. For convenience, the diode stack is cooled and temperature stabilized at $20^{\circ} \mathrm{C}$ by a Peltier module. For the purpose of this study, we kept a constant output energy of $20 \mathrm{~mJ}$ at $1064 \mathrm{~nm}$ for the oscillator. We choose to have this constant energy level to preserve a low M2 parameters for the oscillator $(\mathrm{M} 2<1.2)$. Subsequent amplification in slabs allows us to recover higher energy at the fundamental wavelength before frequency conversion. 


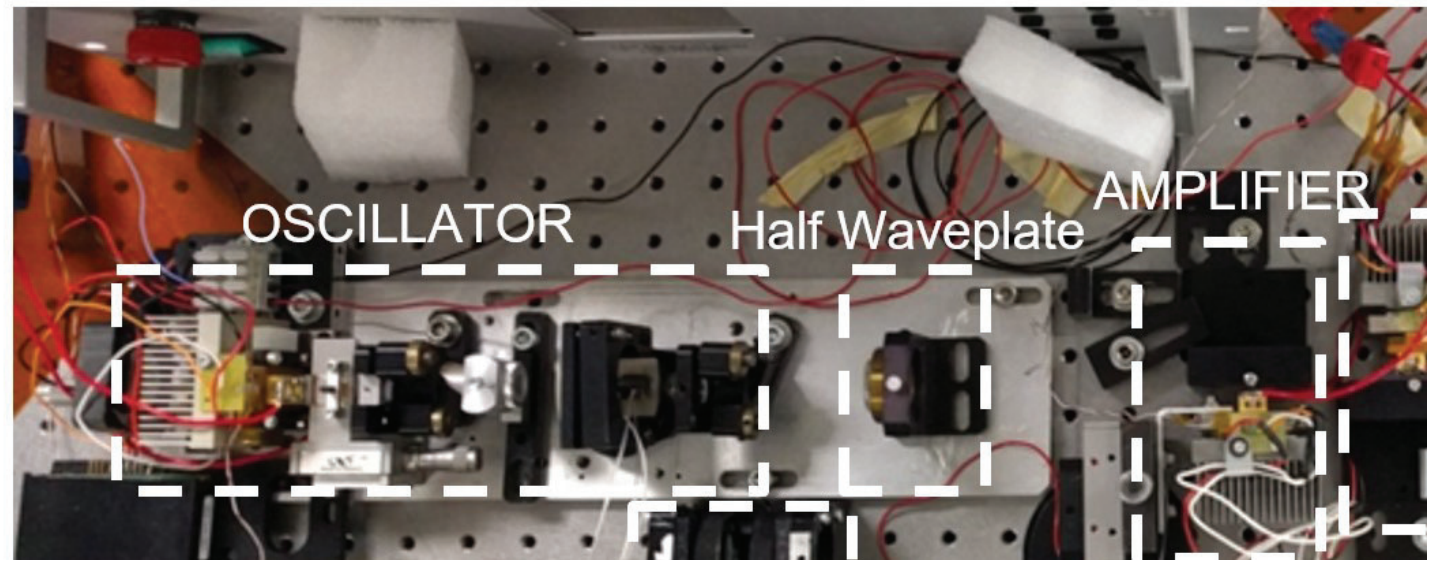

Figure 1. SuperCam test platform on breadboard for harmonic conversion at $355 \mathrm{~nm}$ and $266 \mathrm{~nm}$.

The repetition rate can be set at $3 \mathrm{~Hz}$ or $10 \mathrm{~Hz}$. Following the oscillator, we choose the laser configuration by adding the following modules:

- A beam expander (magnification factor 1.54). We insert the beam expander to lower the peak power density on the slabs when output energy is higher than $80 \mathrm{~mJ}$ (2 amplifiers slab configuration only) to avoid optical damages.

- A half-wave plate to control polarization and optimize harmonic generation. It replaces the RTP Pockels cell used in SuperCam to switch between Raman and LIBS mode

- One or two transversally pumped Nd:YAG slabs. The pumping stack is the same as for the oscillator and is also temperature stabilized at $20^{\circ} \mathrm{C}$

○ Harmonic generation module (see 3.2 and 3.3)

We used dichroic mirrors to separate third or fourth harmonic, $1064 \mathrm{~nm}$ and $532 \mathrm{~nm}$ beams are transmitted while harmonic beam is reflected.

\subsection{Third harmonic generation module}

We selected the following non-linear crystal:

- A KTP (type II) crystal (dimension 6x6x5mm) used also for Second Harmonic Generation in the SuperCam program

- A LBO (Type II) crystal (8x8x12 mm). UV generation with LBO crystals have been done in space program like Aeolus.

Both crystals are mounted on mechanical stages allowing phase matching by rotation of the crystals.

\subsection{Fourth harmonic generation module}

We selected the following non-linear crystal:
- Same KTP crystal
○ A BBO (type I) crystal $(8 \times 8 \times 12 \mathrm{~mm})$.

BBO crystal for FHG was used in program like the MOMA instruments for ExoMars mission. 


\subsection{Measurement set-up}

The measurement set-up includes:

- A joulemeter to measure energy and energy stability

- A beam profiler to measure beam profile in the near and far field. For M2 measurement, the spatial profile is scanned along the beam propagation axis.

- A Si photodiode with $1 \mathrm{~ns}$ rise time for temporal width measurement

We took special care to remove fundamental wavelength and second harmonic generated signals using additional two additional dichroic mirrors and a Pelin Broca prism for spatial and temporal measurements.

\section{EXPERIMENTAL RESULTS}

\subsection{SuperCam test platform at $1064 \mathrm{~nm}$}

The SuperCam laser assembled on a breadboard allows us to test different configurations to increase the energy of the oscillator. We measured energy, pulse duration and beam profile (near field, far field and M2 factor) for each configurations at the fundamental wavelength of the emission of the laser. We summarized results in table 1. All measurements were performed at $3 \mathrm{~Hz}$ except for the 2 slabs and beam expander configuration $(3 \mathrm{~Hz}$ and $10 \mathrm{~Hz})$.

\begin{tabular}{|c|c|c|c|c|}
\hline Laser Configuration & $\begin{array}{c}\text { Oscillator } \\
\text { only }\end{array}$ & 1 slab amplifier & 2 slabs amplifier & $\begin{array}{c}\text { 2 slabs amplifier \& beam } \\
\text { expander (3 or 10 Hz) }\end{array}$ \\
\hline $\begin{array}{c}\text { Maximum Energy } \\
\text { (mJ) }\end{array}$ & 21 & 57 & 81 & 115 \\
\hline $\begin{array}{c}\text { Energy Stability } \\
\text { (RMS \%) }\end{array}$ & 1.4 & 1 & 0.7 & 1 \\
\hline M2 (x axis) & 1.05 & 1.03 & 1 & 1 \\
\hline M2 (y axis) & 1.07 & 1.02 & 1 & 1 \\
\hline
\end{tabular}

Table 1. SuperCam performance at 1064 in four optical configurations.

The spatial profile in the near field and far field is preserved when the beam is amplified in the different configurations.

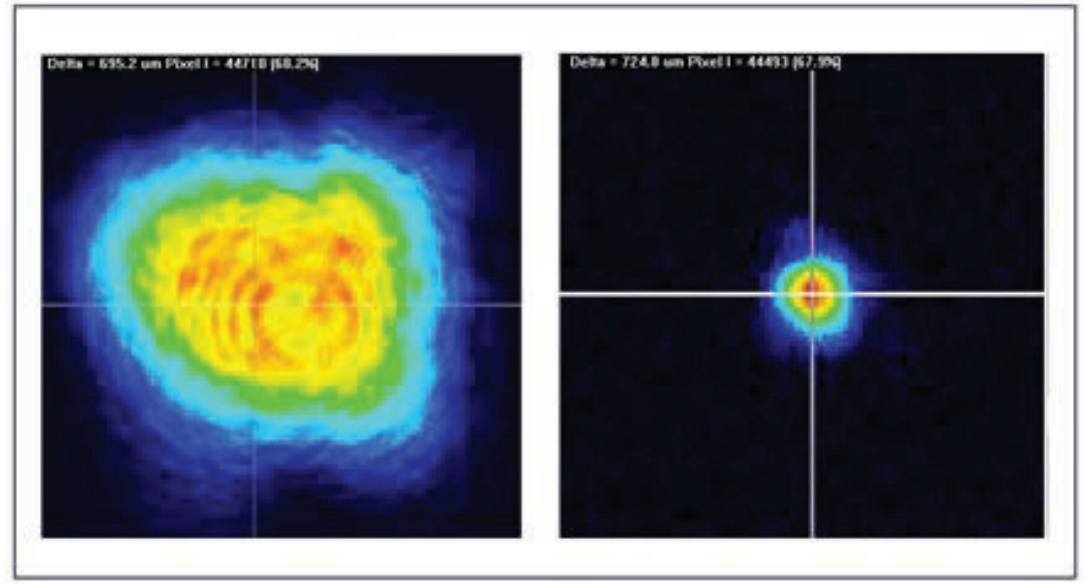

Figure 2. Beam profile at $1064 \mathrm{~nm}$ in the near field (left image) and far field (right image) measured at $3 \mathrm{~Hz}$ for an energy of $115 \mathrm{~mJ}$ ( 2 amplifiers with a beam expander). 
The platform can deliver energy from $20 \mathrm{~mJ}$ (oscillator only) to $115 \mathrm{~mJ}$ (two amplifiers including beam expander). Pulse width (FWHM) is $6 \mathrm{~ns}$ for all configurations.

\subsection{Third Harmonic Generation}

Third harmonic generation for each set-up is optimized by angle tuning of SHG and THG crystals. The highest level in energy of the third harmonic is not obtained for the highest level of second harmonic. Usually, a conversion efficiency of $40 \%$ for the second harmonic is optimal to maximize THG energy output. We have summarized in table the maximum energy achievable for each configuration.

\begin{tabular}{|c|c|c|c|c|}
\hline Laser Configuration & $\begin{array}{c}\text { Oscillator } \\
\text { only }\end{array}$ & 1 slab amplifier & 2 slabs amplifier & $\begin{array}{c}\text { 2 slab amplifier \& beam } \\
\text { expander (3 Hz/10 Hz) }\end{array}$ \\
\hline Maximum energy (mJ) & 5.8 & 16 & 25.3 & $37.4 / 33$ \\
\hline $\begin{array}{c}\text { Energy Stability 50 shots } \\
\text { (RMS \%) }\end{array}$ & 2.5 & 2.5 & 2.2 & $3.8 / 3.5$ \\
\hline $\begin{array}{c}\text { Conversion efficiency } \\
(\%)\end{array}$ & 27.4 & 28 & 31 & $32.5 / 29.2$ \\
\hline M2 x axis & 2 & 1.8 & 1.2 & 1.9 \\
\hline M2 y axis & 1.5 & 1.6 & 1.3 & 1.2 \\
\hline
\end{tabular}

Table 2. SuperCam performance at $355 \mathrm{~nm}$ for 4 different optical configurations.

We can deliver with the various platform tested from $5.8 \mathrm{~mJ}$ at $355 \mathrm{~nm}$ (oscillator only) to $37.4 \mathrm{~mJ}$ at a repetition rate of $3 \mathrm{~Hz}$. Energy stability is better than 2.5\% RMS except for the most energetic configuration (3.8\% RMS for 2 slabs and beam expander). M2 parameter is lower than 2 on both axis for all configurations. FWHM pulse duration is reduced to 5 ns due to temporal filtering in the harmonic generation process.

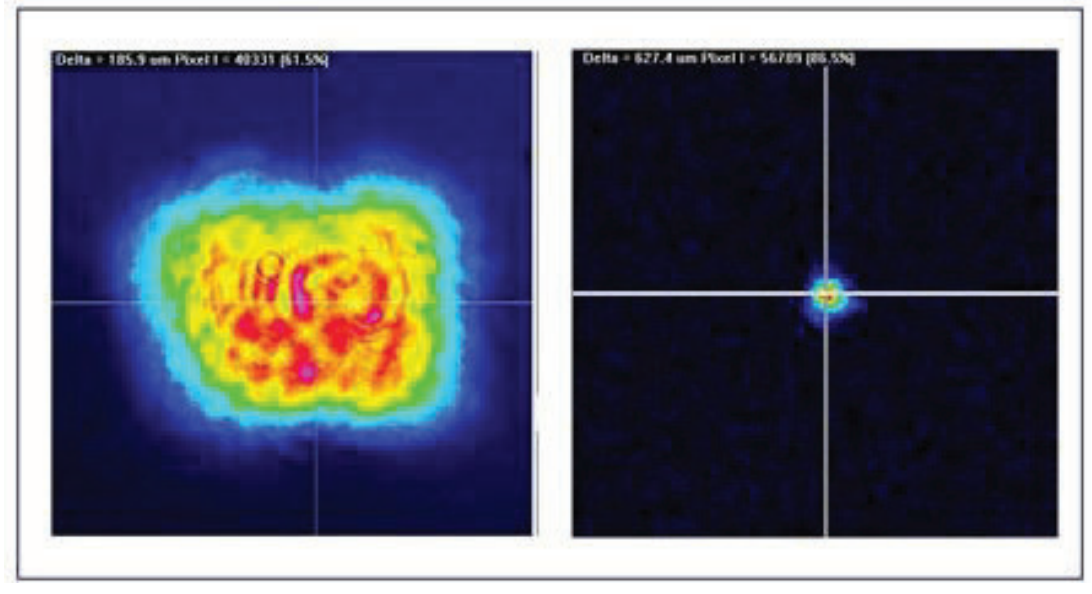

Figure 3. Beam profile at $355 \mathrm{~nm}$ in the near field (left image) and far field (right image) measured at $3 \mathrm{~Hz}$ for an energy of $37 \mathrm{~mJ}$ (2 amplifiers with beam expander).

For the configuration using two slab amplifiers and a beam expander (maximum energy in the fundamental), there is a decrease of converted energy by $10 \%$ when running the laser at $10 \mathrm{~Hz}$ rather than $3 \mathrm{~Hz}$. We attribute this decrease in harmonic conversion efficiency to increased thermal lensing and aberration within the slab amplifiers. 


\subsection{Fourth Harmonic Generation}

Fourth harmonic generation for each set-up is optimized by angle tuning of SHG and FHG crystals. The highest level in energy of the fourth harmonic is obtained for the highest level of second harmonic. We have summarized in table the maximum energy achievable for each configuration.

\begin{tabular}{|c|c|c|c|c|}
\hline Laser Configuration & $\begin{array}{c}\text { Oscillator } \\
\text { only }\end{array}$ & $\begin{array}{c}\text { 1 slab } \\
\text { amplifier }\end{array}$ & 2 slabs amplifier & $\begin{array}{c}\text { 2 slab amplifier \& beam } \\
\text { expander (3 Hz/10Hz) }\end{array}$ \\
\hline Maximum energy (mJ) & 3,1 & 10.9 & 14.9 & $23.4 / 19.7$ \\
\hline $\begin{array}{c}\text { Energy Stability (RMS } \\
\text { \%) }\end{array}$ & 12 & 2.2 & 2.5 & $4.4 /-$ \\
\hline $\begin{array}{c}\text { Conversion efficiency } \\
(\%)\end{array}$ & 14.7 & 19 & 18.4 & 3.4 \\
\hline M2 x axis & 2.6 & 3 & - & 1.7 \\
\hline M2 y axis & 1.7 & 3.4 & - & $20.3 / 17.4$ \\
\hline
\end{tabular}

Table 3. SuperCam performance at $355 \mathrm{~nm}$ for 4 different optical configurations.

Output energy at $266 \mathrm{~nm}$ is $3.1 \mathrm{~mJ}$ for the oscillator and up to $23.4 \mathrm{~mJ}$ for two slabs with the beam expander set-up. Energy stability (12\% RMS) and conversion efficiency are low in the oscillator configuration because of insufficient peak power incident on the BBO crystal. Adding one or two slabs drastically improves energy stability (better than $2.5 \%$ RMS) and conversion efficiency (up to $19 \%$ ) thanks to higher peak power. M2 is lower than 3.4 on both axis in all configurations. Temporal width (FWHM) is measured to be 6ns. We expected shorter duration due to the nonlinear process but are limited by the detector spectral response in the UV.

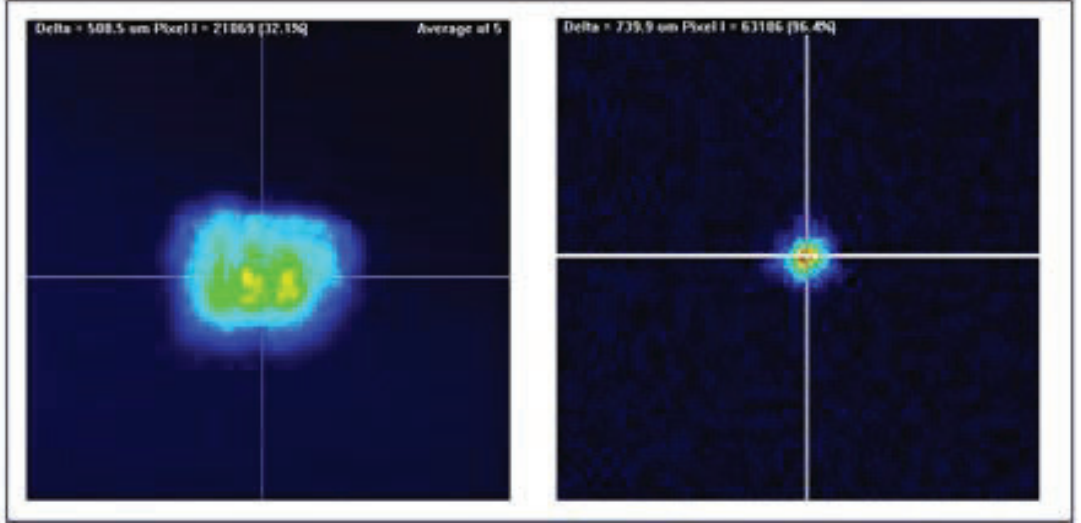

Figure 4. Beam profile at $355 \mathrm{~nm}$ in the near field (left image) and far field (right image) measured at $3 \mathrm{~Hz}$ for an energy of

$37 \mathrm{~mJ}$ ( 2 amplifiers with beam expander).

As for THG, the conversion efficiency drops by $10 \%$ when running at a higher repetition rate. We noticed for all configuration the high sensitivity of angular phase matching of the fourth harmonic generator. In the case of the highest energy version including the beam expander, it is also critical to adjust its divergence precisely to be within the narrow acceptance of the BBO crystal. These constraints could be relaxed by using a shorter BBO crystal (larger acceptance angle) at the cost of a lower harmonic conversion efficiency.

\subsection{Discussion and model design}

From our measurements, we wrote preliminary specifications for a UV laser operating at $355 \mathrm{~nm} \mathrm{or} 266 \mathrm{~nm}$. The designed lasers would be single wavelength, without the capacity to switch between fundamental and UV. Repetition rate is chosen to be $10 \mathrm{~Hz}$ to have a fixed thermal load and hence constant beam divergence during the burst sequence. Mechanical design is herited from SuperCam. Thermal dissipation is purely conductive in the laser (no active cooling 
using Peltier module). Non-linear crystal oven are temperature controlled using heaters as for SuperCam KTP crystal. Additional driving electronics for Q-switch and diodes adds $600 \mathrm{~g}$ to the laser mass.

In table 4, we have summarized performances of both laser versions. Thermal effects experienced during burst shooting were taken into account (thermal dissipation, interplay between diode spectrum and Nd:YAG absorption spectrum) to estimate preliminary performances of the lasers. Different configurations with lower repetition rate and/or lower energy are possible, leading to smaller footprint for the laser.

\begin{tabular}{|c|c|c|}
\hline PARAMETERS & $355 \mathrm{~nm}$ & $266 \mathrm{~nm}$ \\
\hline Repetition rate $(\mathrm{Hz})$ & \multicolumn{2}{|c|}{10} \\
\hline Operation mode & \multicolumn{2}{|c|}{ N shots burst, $1 \leq N \leq 1000$} \\
\hline Pause between burst (min) & \multicolumn{2}{|c|}{$\leq \mathbf{5}$} \\
\hline Average Energy over one burst (mJ) & $\geq 25$ (at one temperature) & $\begin{array}{l}\geq 15 \text { (at one } \\
\text { temperature) }\end{array}$ \\
\hline Pulse length (ns) & \multicolumn{2}{|c|}{$\leq 8$} \\
\hline Wavelength (nm) & $355 \pm 1$ & $266 \pm 1$ \\
\hline M2 & $<\mathbf{3}$ & $<4$ \\
\hline Diameter at $1 / \mathrm{e}^{2}(\mathrm{~mm})$ & \multicolumn{2}{|c|}{$2.5 \leq \mathrm{d} \leq 4$} \\
\hline RMS Energy stability over one burst (\%) & $<8$ & $<12$ \\
\hline $\begin{array}{l}\text { Polarisation } \\
\end{array}$ & \multicolumn{2}{|c|}{ Linearly polarized } \\
\hline Timing jitter Peak to Peak (ns) & \multicolumn{2}{|c|}{$<5$} \\
\hline Mass head(g) & \multicolumn{2}{|c|}{$<800$} \\
\hline Mass electronic (g) & \multicolumn{2}{|c|}{$<600$} \\
\hline Laser Length (mm) & \multicolumn{2}{|c|}{$<330$} \\
\hline Laser Diameter (mm) & \multicolumn{2}{|c|}{$<60$} \\
\hline $\mathrm{T}^{\circ}$ operational range $\left({ }^{\circ} \mathrm{C}\right)$ & \multicolumn{2}{|c|}{+-10 around central $\mathrm{T}^{\circ}$} \\
\hline
\end{tabular}

Table 4. Preliminary specifications for SuperCam type UV model at $355 \mathrm{~nm}$ and $266 \mathrm{~nm}$ operating at $10 \mathrm{~Hz}$.

\section{MINICAM LASER}

In parallel to a UV version of SuperCam, we investigated through CAD simulation a simplified version (MiniCam) for LIBS only application. The idea was to have a smaller/lighter version of SuperCam that can be integrated on a lander or rover smaller than Curiosity/Perseverance class for a potential lunar mission. The performance of the laser in LIBS mode are identical to SuperCam (30 mJ at $1064 \mathrm{~nm}$ ) performance, so the cavity design is kept (see section 2). To minimize risk and keep qualification to a minimum, we decided to use titanium as for ChemCam and SuperCam, and keep laser welding technology to seal the laser. By removing the Raman functionality (Polarization switch and SHG crystal), the laser is shortened and mass is reduced. We also optimized the oscillator structure by adapting geometry and mass repartition. Consequently, we moved the laser collar along the laser axis. The mass for MiniCam is $333 \mathrm{~g}$ (193 g reduction). 

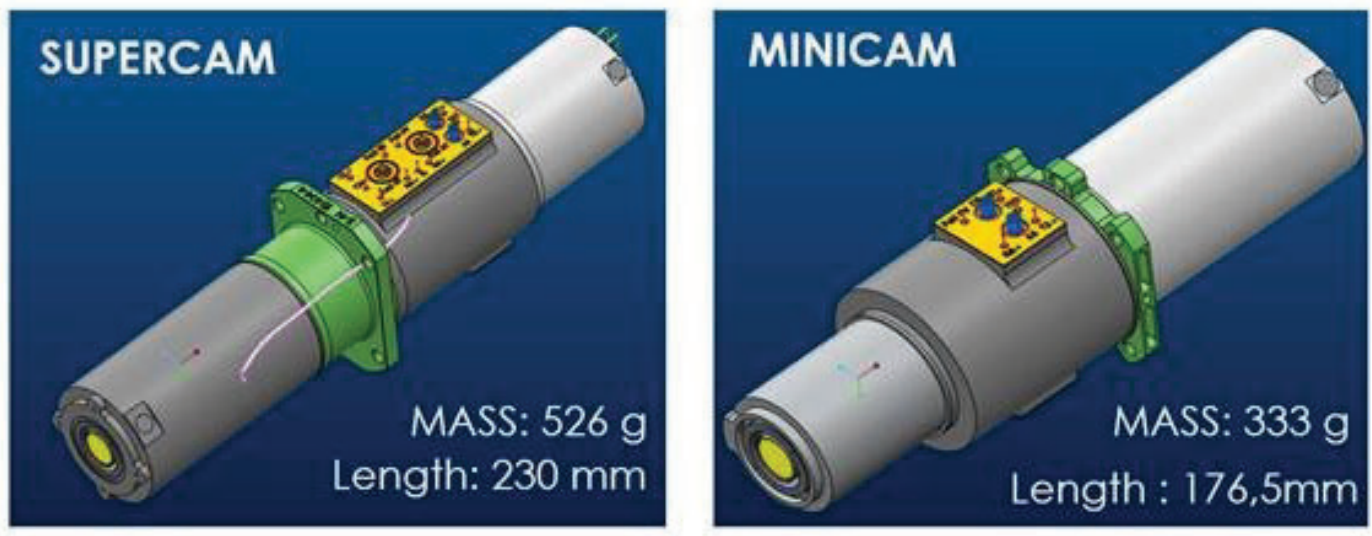

Figure 5. 3D view of SuperCam and MiniCam.

For the electronics of the laser, delivering pulse current to the diode and high voltage to the Q-switch unit, modification were driven by the return of experience on SuperCam. A large margin was kept on SuperCam diode current (230 A for $220 \mu \mathrm{s}$ pulse at $10 \mathrm{~Hz}$ ) in the design phase to ensure energy target (30 mJ for LIBS) could be met over a large temperature range. As the current applied to SuperCam diode stack is never higher than 160 A over the whole qualification temperature range $\left(-40^{\circ} \mathrm{C}\right.$ to $\left.40{ }^{\circ} \mathrm{C}\right)$, we choose this value as the new maximum to be delivered by the electronics. Mass for electronic was reduced from $468 \mathrm{~g}$ to $308 \mathrm{~g}$. Reducing current allowed us to reduce the number of capacitors and surface of the PCB board.

\section{SUMMARY AND OUTLOOK}

We have investigated the performances of UV models emitting at $355 \mathrm{~nm}$ or $266 \mathrm{~nm}$ on SuperCam test platform and estimated potential performances for flight models at the highest energy and repetition rate achievable. We also designed a new version of SuperCam focusing on size reduction for LIBS application.

\section{ACKNOWLEDGEMENT}

The work is funded by the Centre National d'Etudes Spatiales (CNES) under the R\&T grant "Conversion dans l'UV du laser Supercam (R-S18/SU-0003-070)".

\section{REFERENCES}

[1] Forni, O. , "Seven Years of Detection with ChemCam On-Board MSL," Ninth International Conference on Mars, LPI Contribution No.2089, id.6095 (2019)

[2] Durand, E., "Improved conduction cooled compact laser for LIBS -Raman instruments," Proc. SPIE 11180, International Conference on Space Optics, 111802M (2018)

[3] Cosentino, A. , "Spaceborne laser development for ALADIN instrument in inside ESA Aeolus mission," Proc. SPIE 9626, Optical System Design, $96211 \mathrm{U}$ (2015)

[4] Weßels, "UV-DPSS Laser Flight Model for the MOMA Instrument of the ExoMars 2020 Mission," Proc. SPIE 11180, International Conference on Space Optics, 111801B-2 (2018) 